\title{
Enabling Wireless Power Transfer and Multiple Antennas Selection to IoT Network Relying on NOMA
}

\author{
Si-Phu Le ${ }^{1}$, Minh-Sang Van Nguyen ${ }^{2}$, Dinh-Thuan Do $^{3, *}$, Hong-Nhu Nguyen ${ }^{4}$, Ngoc-Long Nguyen ${ }^{4}$, \\ Nhat-Tien Nguyen ${ }^{4}$, Miroslav Voznak ${ }^{4}$ \\ ${ }^{1}$ Van Lang University, \\ Ho Chi Minh City, Vietnam \\ ${ }^{2}$ Faculty of Electronics Technology, Industrial University of Ho Chi Minh City (IUH), \\ Ho Chi Minh City, Vietnam \\ ${ }^{3}$ Department of Computer Science and Information Engineering, \\ College of Information and Electrical Engineering, \\ Asia University, Taichung 41354, Taiwan \\ ${ }^{4}$ VSB Technical University of Ostrava, \\ 17. Listopadu St. 2172/15, 70833 Ostrava-Poruba, Czech Republic \\ dodinhthuan@asia.edu.tw
}

\begin{abstract}
Wireless Power Transfer (WPT) is a significant technique for Internet of Things (IoT) networks. Recently, more interest has been focused on multiple access technique without orthogonal signals for wireless communication. Nonorthogonal Multiple Access (NOMA) scheme is proposed to allow users the access point in IoT network. In this paper, we propose the power beacon which is able to feed energy to power-constraint relay node to further support transmission from the source to destinations in IoT networks. In this article, a NOMA system is benefited by with WPT and antenna selection technique. The system improvement can be achieved through the exact closed-form expressions of outage probability (OP). The performance gap among two users is evaluated using model of the Rayleigh fading channels. Furthermore, we compare NOMA with traditional scheme to highlight advantage of such IoT system.
\end{abstract}

Index Terms - Non-orthogonal multiple access; IoT; Outage probability.

\section{INTRODUCTION}

It has been predicted that Internet will be connected by 50 billion of devices, including small sensors or IoT devices, by 2020 [1]. The fast growth of IoT applications results in requirements of heterogeneous IoT sensor accessing to networks in the upcoming fifth generation (5G) networks. These applications are related to capability of devices in device-to-device (D2D) networks, machine-to-machine (M2M) networks, and other services and applications associated with IoT systems [2]-[5]. It is highly capability to implement the automation in IoT system, thanks to the technological improvement in IoT, and it does not require

Manuscript received 18 April, 2020; accepted 2 September, 2020 The research leading to these results received funding from the Czech Ministry of Education, Youth and Sports under grant No. SP2020/65 conducted at VSB - Technical University of Ostrava. human control [6]. It is necessary to reliable exchange of information and data for these IoT sensors and devices related to the core of distributed automation [7]. An enormous amount of power is consumed by these small sensors and IoT devices to serve their data transmission and communication. However, popular networks contain these battery-powered small sensors or battery-powered devices. It is noted that there is higher demand to remain operation of huge number of sensors, especially providing selfsustainable green communications for IoT networks since devices are limited energy [8]. These power-constraint sensors need their lifetime to be extended, and hence it requires possible solution of energy efficient data transmission.

Unfortunately, it is difficult to replace large numbers of sensors in IoT systems. As a result, the power-constraint devices are operated in practice, and such situation limits the performance improvement. The energy harvesting technique is proposed to tackle this problem by harvesting the energy from the surrounding environments. To harvest the energy from the radio-frequency signals, Radio-frequency (RF) energy harvesting is applied [9]. The relaying networks have been widely studied by introducing flexible, sustainable, and stable energy supply to devices in such networks [10]-[14]. In [11], renewable energy is considered as a solution to employ dense small cell base stations (SBSs) to adapt to the increasing demand of communication services. The authors in [12] studied for unmanned aerial vehicle (UAV)-assisted networks in term of the resource allocation problem. In this system, multiple energy harvesting-powered D2D pairs are powered by a UAV which play UAV as an energy source providing radio-frequency energy. In [13], system throughput can be enhanced by utilizing optimal channel selection method and the harvested RF energy as well. The 
cognitive radio sensor networks benefit from the RF energy harvesting in [14].

Considering as a prominent wireless access technique for the $5 \mathrm{G}$ wireless communication, Non-orthogonal Multiple Access (NOMA) is introduced and analysed in [15]. To provide higher spectrum efficiency, NOMA employs nonorthogonal transmission at the transmitter. The transmitter divides power domain to serve multiple users with superposed signal transmitted. Different from Orthogonal Multiple Access (OMA), NOMA can serve multiple users over the same resource block, thus it can effectively improve sum rate in other emerging networks [16], [17]. To decode the users' information at the receiver, the Successive Interference Cancellation (SIC) is required. Specifically, signal is decoded firstly for the user with the best channel condition while assuming other users' signal as interference. However, results in [16] did not consider multiple antennas and multiple beacon since these techniques benefit to performance improvement. The IoT benefits from power beacon, and some metrics are studied to exhibit system performance [18]. Motivated by these papers [16-18], we formulate the problem of selection of beacon and antenna selection to evaluate outage performance of two NOMA users.

The remaining parts of this paper are arranged as follows. Section II presents the system model based on NOMA to implement IoT system. In Section III, we consider the outage performance of such NOMA applied together with Wireless Power Transfer (WPT). In addition, the traditional technique of OMA is presented in Section IV. The numerical simulations are conducted in Section $\mathrm{V}$, and we provide conclusion remarks in Section VI.

\section{SYSTEM MODEL}

In this model, Fig. 1 shows the IoT system containing the access point $(A P)$, the relay $R$, two users $D_{1}, D_{2}$, and power beacon $(B)$. Wireless channels denoted as in Fig. 1 in such IoT system relying NOMA are subjected to Rayleigh flat fading plus additive white Gaussian noise. The complex channel coefficients for the links $A P \rightarrow R, \quad B \rightarrow R$, $R \rightarrow D_{1}, \quad R \rightarrow D_{2}$ are represented by $\left|h_{S_{k} R}\right|^{2} \sim C N\left(0, \lambda_{S R}\right)$, $\left|h_{B R}\right|^{2} \sim C N\left(0, \lambda_{B R}\right), \quad\left|h_{R D_{1}}\right|^{2} \sim C N\left(0, \lambda_{R D 1}\right), \quad$ and $\left|h_{R D_{2}}\right|^{2} \sim C N\left(0, \lambda_{R D 2}\right)$, respectively. There are $K$ antennas equipped at the $A P$.

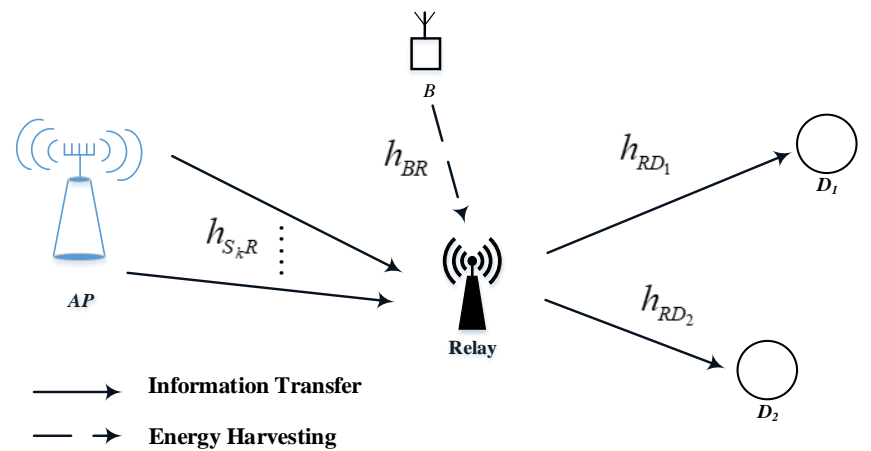

Fig. 1. System Model of multiple power beacon-assisted NOMA.
There are two links from the $A P$ to NOMA users. In the first phase, the received signal at the relay is given by

$$
y_{B S-R}=\sqrt{P_{B S}} h_{S_{k} R}\left(\sqrt{a_{1}} x_{1}+\sqrt{a_{2}} x_{2}\right)+\omega_{R},
$$

where $P_{B S}$ is the transmit power of $A P, x_{i}, i \in\{1,2\}$ is the information symbol for $D_{i} . a_{i}$ is the power allocation coefficient for $x_{i}$ with $a_{1}+a_{2}=1$ and $a_{1}>a_{2}$, and $\omega_{R}$ is the Additive White Gaussian Noise (AWGN) at the relay. Optimization of such power allocation is out of concern in this paper.

It can be achieved by the signal to interference plus noise ratio (SINR) at the receivers. In order to decode $x_{1}$ at $R$, the corresponding SINR is expressed by

$$
\gamma_{S_{k} R-x_{1}}=\frac{a_{1} P_{B S}\left|h_{S_{k} R}\right|^{2}}{a_{2} P_{B S}\left|h_{S_{k} R}\right|^{2}+N_{0}} .
$$

In NOMA, SIC is employed to eliminate interference, the SINR to decode $x_{2}$ is given by

$$
\gamma_{S_{k} R-x_{2}}=\frac{a_{2} P_{B S}\left|h_{S_{k} R}\right|^{2}}{N_{0}} .
$$

During time for signal processing in the second phase, $R$ transmits the signal consisting of the decoded and reencoded symbols to the destinations. The received signal at two users $D_{i}$ is given by

$$
y_{R-D_{i}}=\sqrt{P_{R}} h_{R D_{i}}\left(\sqrt{a_{1}} x_{1}+\sqrt{a_{2}} x_{2}\right)+\omega_{D_{i}}
$$

where $P_{R}$ is the transmit power of $R, \omega_{D_{i}}$ is the AWGN at $D_{i}$.

The signal to interference plus noise ratio (SINR) at each receiver needs to be calculated. In this case, the destination is required to decode $x_{1}$ at $D_{1}$ as below

$$
\gamma_{D_{1}-x_{1}}=\frac{a_{1} P_{R}\left|h_{R D_{1}}\right|^{2}}{a_{2} P_{R}\left|h_{R D_{1}}\right|^{2}+N_{0}} .
$$

The SINR to decode $x_{1}$ at $D_{2}$ is given by

$$
\gamma_{D_{2}-x_{1}}=\frac{a_{1} P_{R}\left|h_{R D_{2}}\right|^{2}}{a_{2} P_{R}\left|h_{R D_{2}}\right|^{2}+N_{0}} .
$$

After SIC, the SINR to decode $x_{2}$ is given by

$$
\gamma_{D_{2}-x_{2}}=\frac{a_{2} P_{R}\left|h_{R D_{2}}\right|^{2}}{N_{0}} .
$$

The best channel is selected with the index of antennas 
equipped at the $A P$ as

$$
k^{*}=\arg \max _{k=1, \ldots, K}\left(\left|h_{S_{k} R}\right|^{2}\right) .
$$

Together with (8), the cumulative distribution function (CDF) and probability distribution function (PDF) related to selected channels are given as

$$
F_{\left|h_{S_{k} * R}\right|^{2}}(x)=1-\sum_{k=1}^{K}\left(\begin{array}{l}
K \\
k
\end{array}\right)(-1)^{k-1} \exp \left(-\frac{k x}{\lambda_{S R}}\right),
$$

and

$$
f_{\mid h_{\left.S_{k^{*} R}\right|^{2}}}(x)=\sum_{k=1}^{K}\left(\begin{array}{l}
K \\
k
\end{array}\right)(-1)^{k-1} \frac{k}{\lambda_{S R}} \exp \left(-\frac{k x}{\lambda_{S R}}\right) \text {. }
$$

In the considered system, the relay harvests energy from beacons. The operation of the second stage of signal processing is supported by harvests energy at relay.

At energy harvesting phase, the time switching (TS) based energy harvesting technique is applied. In a transmission block time $T$ (in which a block of information is sent from the beacon to the relay), the relay takes $\alpha T$ to harvest energy from the beacon, in which $\alpha$ is the energy harvesting time fraction that depends on the schedule of $B$. We allocate the time slot of $(1-\alpha) T$ into two equal sub-time slots for the link from the $A P$ to relay * and the link relaydestinations. Then, we can compute harvested energy at the relay as in [10]

$$
E_{R}=\tau P_{B} \alpha T\left|h_{B R}\right|^{2},
$$

where $0<\tau<1$ stands for the efficiency coefficient of the energy conversion process, $0<\alpha<1$ is the percentage of energy harvesting, $P_{B}$ is the transmit power of the beacon, assuming that these power beacons have the same power level, respectively (optimizing time switching factor $\alpha$ is out of the scope of this paper). Under the assumption that the processing energy at $R$ is negligible, the transmit power of the relay is

$$
P_{R}=\frac{2 \tau P_{B} \alpha\left|h_{B R}\right|^{2}}{(1-\alpha)}=\xi\left|h_{B R}\right|^{2},
$$

where $\xi=\frac{2 \tau P_{B} \alpha}{(1-\alpha)}$

\section{OUtAge PERFormance ANALYSIS FOR NOMA}

In this section, we consider the outage probability analyses for the IoT system to look for impact of harvested energy and the number of transmit antennas at the $A P$. In particular, we derive the closed-form expressions to show the outage probabilities, and performance difference happens as comparing two users' performance. To provide insights, asymptotic outage performance analyses for the considered system are determined in the high transmit signal to noise ratio (SNR) region.

\section{A. Outage Analysis at $D_{1}$}

With respect to the system performance evaluation, the outage probabilities can be achieved related to ability to detect signal at relay and destinations as well. The expression of outage probability for the first user can be defined as

$$
\begin{gathered}
O P_{1}=\operatorname{Pr}\left(\min \left(\gamma_{S_{k^{*} R-x_{1}}}, \gamma_{D_{1}-x_{1}}\right)<v_{1}\right)= \\
=1-\operatorname{Pr}\left(\gamma_{S_{k^{*} R-x_{1}}}>v_{1}, \gamma_{D_{1}-x_{1}}>v_{1}\right)= \\
=1-\underbrace{\operatorname{Pr}\left(\gamma_{S_{k^{*} R-x_{1}}}>v_{1}\right)}_{\partial_{1}} \times \underbrace{\operatorname{Pr}\left(\gamma_{D_{1}-x_{1}}>v_{1}\right)}_{\partial_{2}},
\end{gathered}
$$

where $v_{i}=2^{2 R_{i}}-1, \quad R_{i}$ is the target rate to decode $x_{i}$, $i=\{1,2\}$.

Proposition 1: The close-form expression is computed to provide outage analysis at $D_{1}$ as

$$
\begin{gathered}
O P_{1}=1-\sum_{k=1}^{K}\left(\begin{array}{l}
K \\
k
\end{array}\right)(-1)^{k-1} \exp \left(-\frac{k v_{1} N_{0}}{P_{B S}\left(a_{1}-v_{1} a_{2}\right) \lambda_{S R}}\right) \times \\
\times \sqrt{\frac{4 v_{1} N_{0}}{\xi\left(a_{1}-v_{1} a_{2}\right) \lambda_{B R} \lambda_{R D 1}}} K_{1}\left(\sqrt{\frac{4 v_{1} N_{0}}{\xi\left(a_{1}-v_{1} a_{2}\right) \lambda_{B R} \lambda_{R D 1}}}\right) .
\end{gathered}
$$

where $K_{1}($.$) is the second kind of modified Bessel$ functions.

Proof: Please refer to Appendix A.

\section{B. Outage Analysis at $\mathrm{D}_{2}$ with Perfect SIC}

At the second user, it can be seen outage performance evaluated by

$$
\begin{aligned}
& O P_{2}=\operatorname{Pr}\left(\min \left(\gamma_{k_{k^{*}} R-x_{2}}, \gamma_{D_{2}-x_{1}}, \gamma_{D_{2}-x_{2}}\right)<v_{2}\right)= \\
= & 1-\operatorname{Pr}\left(\gamma_{{k^{*}}^{*} R-x_{2}}>v_{2}, \gamma_{D_{2}-x_{1}}>v_{2}, \gamma_{D_{2}-x_{2}}>v_{2}\right)= \\
= & 1-\underbrace{\operatorname{Pr}\left(\gamma_{{k^{*}}^{*} R-x_{2}}>v_{2}\right)}_{\nabla_{1}} \times \underbrace{\operatorname{Pr}\left(\gamma_{D_{2}-x_{1}}>v_{2}, \gamma_{D_{2}-x_{2}}>v_{2}\right)}_{\nabla_{2}} .
\end{aligned}
$$

Proposition 2: It can be achieved the close-form expression to provide outage analysis perfect SIC at $D_{2}$ in perfect SIC case as

$$
\begin{aligned}
O P_{2} & =1-\sum_{k=1}^{K}\left(\begin{array}{l}
K \\
k
\end{array}\right)(-1)^{k-1} \exp \left(-\frac{k v_{2} N_{0}}{a_{2} P_{B S} \lambda_{S R}}\right) \times \\
& \times \sqrt{\frac{4 \theta v_{2} N_{0}}{\xi \lambda_{B R} \lambda_{R D 2}}} K_{1}\left(\sqrt{\frac{4 \theta v_{2} N_{0}}{\xi \lambda_{B R} \lambda_{R D 2}}}\right) .
\end{aligned}
$$

\section{Proof: Please refer to Appendix B.}

\section{Scenario of Imperfect SIC}

The SINR to decode $x_{2}$ at the first link $A P$-relay is given by 


$$
\gamma_{S_{k} R i p-x_{2}}=\frac{a_{2} P_{B S}\left|h_{S_{k} R}\right|^{2}}{a_{1} P_{B S}\left|g_{S_{k} R}\right|^{2}+N_{0}},
$$

where $g_{S_{k} R} \sim C N\left(0, \mu \lambda_{\text {SRip }}\right)$ and $\mu$ is denoted as the level of residual interference caused by imperfect SIC as $0 \leq \mu \leq 1$.

The second link is evaluated via SINR as below. It is used to decode $x_{2} D_{2}$ and such SINR is formulated by

$$
\gamma_{D_{2} i p-x_{2}}=\frac{a_{2} P_{R}\left|h_{R D_{2}}\right|^{2}}{a_{1} P_{R}\left|g_{R D_{2}}\right|^{2}+N_{0}},
$$

where $g_{R D_{2}} \sim C N\left(0, \mu \lambda_{R D 2 i p}\right)$.

The outage performance of user $D_{2}$ in imperfect SIC case can be written as

$$
\begin{gathered}
O P_{2 i p}=\operatorname{Pr}\left(\min \left(\gamma_{S_{k^{*}} R i p-x_{2}}, \gamma_{D_{2} i p-x_{2}}\right)<v_{2}\right)= \\
=1-\operatorname{Pr}\left(\gamma_{S_{k^{*} R i p-x_{2}}}>v_{2}, \gamma_{D_{2} i p-x_{2}}>v_{2}\right)= \\
=1-\underbrace{\operatorname{Pr}\left(\gamma_{S^{*} R i p-x_{2}}>v_{2}\right)}_{\psi_{1}} \times \underbrace{\operatorname{Pr}\left(\gamma_{D_{2} i p-x_{2}}>v_{2}\right)}_{\psi_{2}} .
\end{gathered}
$$

Proposition 3: The closed-form in outage analysis at $D_{2}$ is formulated (imperfect SIC case) by

$$
\begin{gathered}
O P_{2 i p}=1-\sum_{k=1}^{K} \sum_{m=1}^{K}\left(\begin{array}{l}
K \\
k
\end{array}\right)\left(\begin{array}{l}
K \\
m
\end{array}\right)(-1)^{k+m-2} \times \\
\times \frac{m a_{2} \lambda_{S R}}{k v_{2} a_{1} \lambda_{S R i p}+m a_{2} \lambda_{S R}} \exp \left(-\frac{k v_{2} N_{0}}{a_{2} P_{B S} \lambda_{S R}}\right) \times \\
\times \frac{a_{2} \xi \lambda_{R D 2}}{v_{2} a_{1} \xi \lambda_{R D 2 i p}+a_{2} \xi \lambda_{R D 2}} \times \\
\times \sqrt{\frac{4 v_{2} N_{0}}{a_{2} \xi \lambda_{R D 2} \lambda_{B R}}} K_{1}\left(\sqrt{\frac{4 v_{2} N_{0}}{a_{2} \xi \lambda_{R D 2} \lambda_{B R}}}\right) .
\end{gathered}
$$

Proof: Please refer to Appendix C.

\section{Asymptotic Outage Probability Analysis}

When $P_{B S} \rightarrow \infty$, asymptotic performance of $D_{1}$ and $D_{2}$. can be obtained. We first look on the asymptotic performance of the first user in terms of outage probability as:

$$
\begin{gathered}
O P_{1}^{a s y m}=1-\sqrt{\frac{4 v_{1} N_{0}}{\xi\left(a_{1}-v_{1} a_{2}\right) \lambda_{B R} \lambda_{R D 1}}} \times \\
\times K_{1}\left(\sqrt{\frac{4 v_{1} N_{0}}{\xi\left(a_{1}-v_{1} a_{2}\right) \lambda_{B R} \lambda_{R D 1}}}\right), \\
O P_{2}^{a s y m}=1-\sqrt{\frac{4 \theta v_{2} N_{0}}{\xi \lambda_{B R} \lambda_{R D 2}}} K_{1}\left(\sqrt{\frac{4 \theta v_{2} N_{0}}{\xi \lambda_{B R} \lambda_{R D 2}}}\right) .
\end{gathered}
$$

It is worth noting that perfect SIC and imperfect SIC cases are considered for user $D_{2}$, respectively, as below

$$
\begin{gathered}
O P_{2 i p}^{a s y m}=1-\sum_{k=1}^{K} \sum_{m=1}^{K}\left(\begin{array}{l}
K \\
k
\end{array}\right)\left(\begin{array}{l}
K \\
m
\end{array}\right)(-1)^{k+m-2} \times \\
\times \frac{m a_{2} \lambda_{S R}}{k v_{2} a_{1} \lambda_{S R i p}+m a_{2} \lambda_{S R}} \frac{a_{2} \xi \lambda_{R D 2}}{v_{2} a_{1} \xi \lambda_{R D 2 i p}+a_{2} \xi \lambda_{R D 2}} \times \\
\times \sqrt{\frac{4 v_{2} N_{0}}{a_{2} \xi \lambda_{R D 2} \lambda_{B R}}} K_{1}\left(\sqrt{\frac{4 v_{2} N_{0}}{a_{2} \xi \lambda_{R D 2} \lambda_{B R}}}\right) .
\end{gathered}
$$

\section{OUTAGE PERFormanCE OF OMA}

In the first phase, the received signal at the first link $A P$ relay is given by

$$
y_{B S-R}^{O M A}=\sqrt{P_{B S}} h_{S_{k} R} x_{i}+\omega_{R} .
$$

The SINR computed to decode $x_{i}$ at $R$ is given by

$$
\gamma_{S_{k} R-x_{i}}^{O M A}=\frac{P_{B S}\left|h_{S_{k} R}\right|^{2}}{N_{0}} .
$$

After that, $R$ transmits the mixture signal (the decoded and re-encoded symbols) to the destinations. The received signal at $D_{i}$ is given by

$$
y_{R-D_{i}}^{O M A}=\sqrt{P_{R}} h_{R D_{i}} x_{i}+\omega_{D_{i}} .
$$

The SINR to decode $x_{i}$ at $D_{i}$ is given by

$$
\gamma_{D_{i}-x_{i}}^{O M A}=\frac{P_{R}\left|h_{R D_{i}}\right|^{2}}{N_{0}} .
$$

The outage probability at $D_{i}$ can be expressed as

$$
\begin{gathered}
O P_{i}^{O M A}=\operatorname{Pr}\left(\min \left(\gamma_{S_{k^{*}} R-x_{i}}^{O M A}, \gamma_{D_{i}-x_{i}}^{O M A}\right)<v_{i}^{O M A}\right)= \\
=1-\operatorname{Pr}\left(\gamma_{S^{*} R-x_{i}}^{O M A}>v_{i}^{O M A}\right) \times \operatorname{Pr}\left(\gamma_{D_{i}-x_{i}}^{O M A}>v_{i}^{O M A}\right)= \\
=1-\operatorname{Pr}\left(\left|h_{S_{k^{*}} \mid}\right|^{2}>\frac{v_{i}^{O M A} N_{0}}{P_{B S}}\right) \times \operatorname{Pr}\left(\left|h_{B R}\right|^{2}>\frac{v_{i}^{O M A} N_{0}}{\xi\left|h_{R D_{i}}\right|^{2}}\right)= \\
=1-\sum_{k=1}^{K}\left(\begin{array}{l}
K \\
k
\end{array}\right)(-1)^{k-1} \frac{1}{\lambda_{R D i}} \exp \left(-\frac{k v_{i}^{O M A} N_{0}}{P_{B S} \lambda_{S R}}\right) \times \\
\times \int_{0}^{\infty} \exp \left(-\frac{v_{i}^{O M A} N_{0}}{\xi x}-\frac{x}{\lambda_{R D i}}\right) d x= \\
=1-\sum_{k=1}^{K}\left(\begin{array}{l}
K \\
k
\end{array}\right)(-1)^{k-1} \exp \left(-\frac{k v_{i}^{O M A} N_{0}}{P_{B S} \lambda_{S R}}\right) \times \\
\times \sqrt{\frac{4 v_{i}^{O M A} N_{0}}{\xi \lambda_{R D i}}} K_{1}\left(\sqrt{\frac{4 v_{i}^{O M A} N_{0}}{\xi \lambda_{R D i}}}\right),
\end{gathered}
$$

where $v_{i}^{O M A}=2^{4 R_{i}}-1$. 


\section{NUMERICAL RESULTS}

In this section, we show the comparisons of IoT system related outage performance of two users using NOMA and OMA. These users are grouped in downlink of the $A P$ using Rayleigh fading channels under different simulated parameters.

The outage probability versus the transmit SNR at the $A P$ is illustrated in Fig. 2, where we consider two main scenarios, i.e., NOMA and OMA. The different power allocation coefficients are assigned to two users, and hence outage performance of the first user is better than that of the second user. It can be easily seen that more antennas result in lowest outage. When the SNR is greater than $30(\mathrm{~dB})$, outage probabilities for these cases go to straight line. It means that they meet saturation situation.

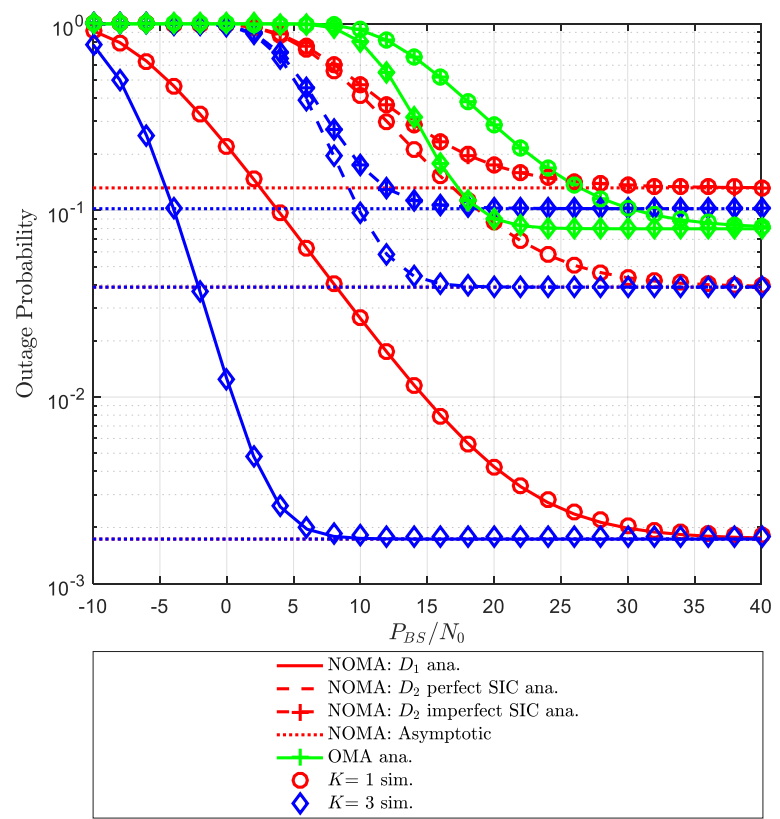

Fig. 2. Outage performance comparison of $D_{1}, D_{2}$ of NOMA and OMA versus $\quad P_{B S} / N_{0} \quad$ by varying $K \quad\left(a_{1}=0.7, \quad \tau=0.6, \quad \alpha=0.5\right.$, $R_{1}=0.5, R_{2}=2, \quad \lambda_{S P}=\lambda_{B R}=1, \quad \lambda_{S R}=\lambda_{R D 1}=10, \quad \lambda_{R D 2}=5$, $\left.\lambda_{\text {SRip }}=\lambda_{\text {RD2ip }}=0.01, \quad P_{B} / N_{0}=30(\mathrm{~dB})\right)$.

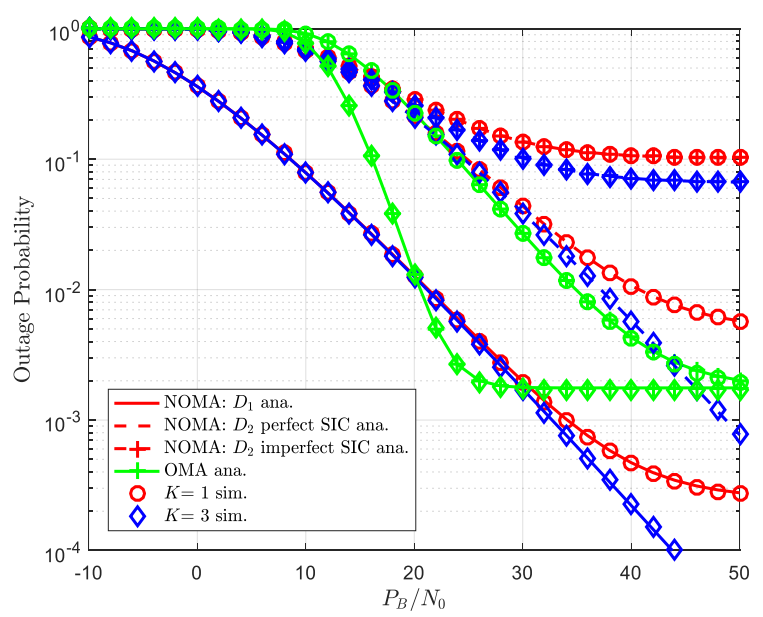

Fig. 3. Outage performance comparison of $D_{1}, D_{2}$ of NOMA and OMA versus $P_{B} / N_{0} \quad$ by varying $K \quad\left(a_{1}=0.7, \quad \tau=0.6, \quad \alpha=0.5\right.$, $R_{1}=0.5, R_{2}=2, \quad \lambda_{S P}=\lambda_{B R}=1, \quad \lambda_{S R}=\lambda_{R D 1}=10, \quad \lambda_{R D 2}=5$, $\left.\lambda_{\text {SRip }}=\lambda_{\text {RD2ip }}=0.01, \quad P_{B S} / N_{0}=30(\mathrm{~dB})\right)$.
In addition, imperfect SIC at the second user has worse outage performance compared with perfect case. It is further confirmed that NOMA in IoT is better than in OMA case. The exactness of the asymptotic lines corresponding with derived expressions for all the considered cases is confirmed at high SNR. Similar trend can be seen in Fig. 3 as considering impact of transmit SNR at the power beacon on the outage probability.

Considering outage performance of two users versus transmit SNR at the $A P$ with different power allocation factors as in Fig. 4, the users' performance change based on the amount of power allocated. Higher $a_{1}$ leads to better outage performance at the first user. These trends of curves related outage behavior are similar as in Fig. 2 and Fig. 3. While considering how transmit SNR at beacon makes impact on outage probability, it can be seen similar performance as in Fig. 5.

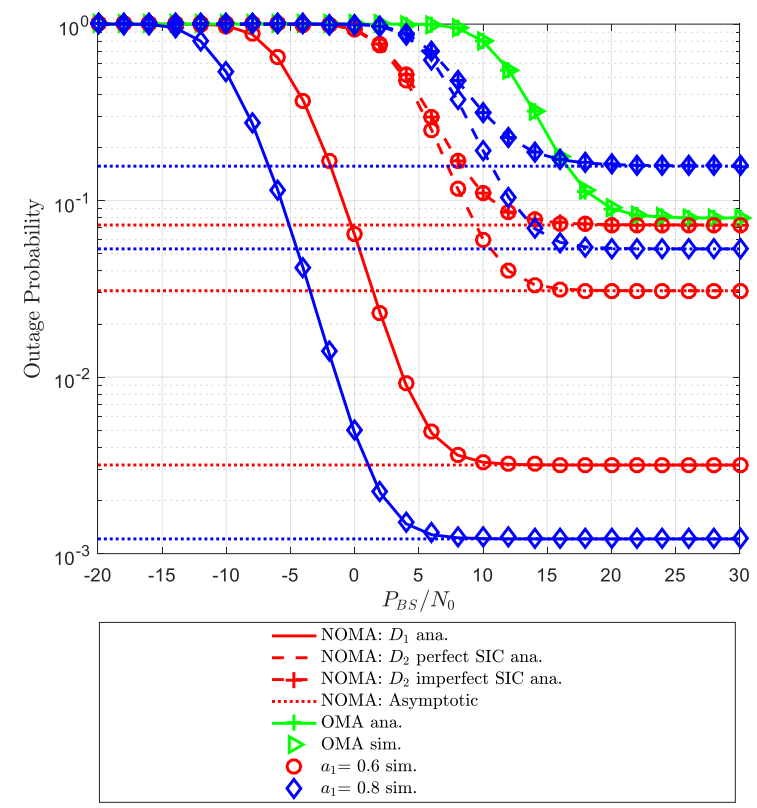

Fig. 4. Outage performance comparison of $D_{1}, D_{2}$ for NOMA and OMA versus $P_{B S} / N_{0}$ by varying $a_{1} \quad\left(\tau=0.6, \quad \alpha=0.5, \quad R_{1}=0.5, R_{2}=2\right.$, $\lambda_{S P}=\lambda_{B R}=1, \quad \lambda_{S R}=\lambda_{R D 1}=10, \quad \lambda_{R D 2}=5, \quad \lambda_{S R i p}=\lambda_{R D 2 i p}=0.01$, $\left.P_{B} / N_{0}=30(\mathrm{~dB}), \quad K=3\right)$.

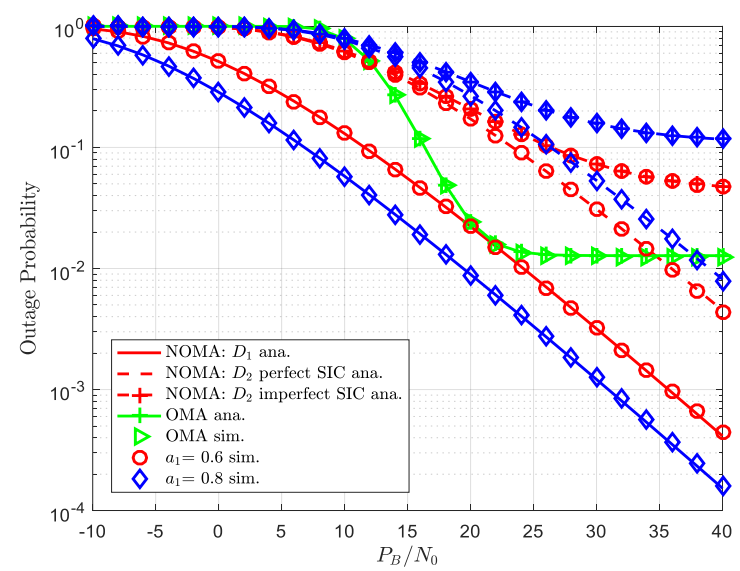

Fig. 5. Outage performance comparison of $D_{1}, D_{2}$ of NOMA and OMA versus $P_{B} / N_{0} \quad$ by varying $a_{1} \quad\left(\tau=0.6, \quad \alpha=0.5, R_{1}=0.5, \quad R_{2}=2\right.$, $\lambda_{S P}=\lambda_{B R}=1, \quad \lambda_{S R}=\lambda_{R D 1}=10, \quad \lambda_{R D 2}=5, \quad \lambda_{S R i p}=\lambda_{R D 2 i p}=0.01$, $\left.P_{B S} / N_{0}=30(\mathrm{~dB}), \quad K=3\right)$. 


\section{CONCLUSIONS}

In this paper, we have investigated the IoT system by enabling energy harvesting and transmit antenna selection schemes. The main result of NOMA scheme provide acceptable outage performance. Such performance is improved significantly at high SNR regime. The relaying scheme with WPT technique benefits to such IoT with performance improvement for two far users who need assistance of WPT-assisted relay. Depending on power allocation factors, different performance of two users can be observed. When SIC can be operated perfectly, it is able to exhibit better performance for the second user. For the antenna selection scheme, it is unnecessary design of multiple antennas system with complex signal processing technique, it can be reduced by exploiting antenna selection as presented in this paper. We derived in closed-form for the outage probability for two distant users in the considered IoT system. More importantly, the asymptotic expressions for the outage probabilities are provided. The superior outage performance achieved by the proposed IoT system is confirmed in numerical results.

\section{APPENDIX A}

Proof of the Proposition 1:

By using (9) and (13), $\partial_{1}$ can be formulated by

$$
\begin{gathered}
\partial_{1}=\operatorname{Pr}\left(\gamma_{S_{k^{*} R-x_{1}}}>v_{1}\right)= \\
=\operatorname{Pr}\left(\left|h_{S_{k^{*} R}}\right|^{2}>\frac{v_{1} N_{0}}{P_{B S}\left(a_{1}-v_{1} a_{2}\right)}\right)= \\
=\sum_{k=1}^{K}\left(\begin{array}{l}
K \\
k
\end{array}\right)(-1)^{k-1} \exp \left(-\frac{k v_{1} N_{0}}{P_{B S}\left(a_{1}-v_{1} a_{2}\right) \lambda_{S R}}\right) .
\end{gathered}
$$

In similar way, from (10) and (13), it can be obtained $\partial_{2}$ as

$$
\begin{gathered}
\partial_{2}=\operatorname{Pr}\left(\gamma_{D_{1}-x_{1}}>v_{1}\right)= \\
=\operatorname{Pr}\left(\left|h_{R D_{1}}\right|^{2}>\frac{v_{1} N_{0}}{\left|h_{B R}\right|^{2} \xi\left(a_{1}-v_{1} a_{2}\right)}\right)= \\
=\int_{0}^{\infty} \exp \left(-\frac{v_{1} N_{0}}{\xi\left(a_{1}-v_{1} a_{2}\right) \lambda_{R D 1} x}\right) \frac{1}{\lambda_{B R}} \exp \left(-\frac{x}{\lambda_{B R}}\right) d x= \\
=\frac{1}{\lambda_{B R}} \int_{0}^{\infty} \exp \left(-\frac{v_{1} N_{0}}{\xi\left(a_{1}-v_{1} a_{2}\right) \lambda_{R D 1} x}-\frac{x}{\lambda_{B R}}\right) d x= \\
=\sqrt{\frac{4 v_{1} N_{0}}{\xi\left(a_{1}-v_{1} a_{2}\right) \lambda_{B R} \lambda_{R D 1}} K_{1}\left(\sqrt{\frac{4 v_{1} N_{0}}{\xi\left(a_{1}-v_{1} a_{2}\right) \lambda_{B R} \lambda_{R D 1}}}\right) .}
\end{gathered}
$$

It is worth noting that the last equation follows from the fact that $\int_{0}^{\infty} \exp \left(-\frac{\delta}{4 x}-\varphi x\right) d x=\sqrt{\frac{\delta}{\varphi}} K_{1}(\sqrt{\delta \varphi})$ in [19, eq. (3.324)].

If we plug (A.1), (A.2) into (13), $O P_{1}$ as the proposition can be achieved.

This is end of the proof.

\section{APPENDIX B}

Proof of the Proposition 2:

Using (9) and (15), $\nabla_{1}$ can be computed as below

$$
\begin{gathered}
\nabla_{1}=\operatorname{Pr}\left(\gamma_{S_{k^{*} R-x_{2}}}>v_{2}\right)= \\
=\operatorname{Pr}\left(\left|h_{S_{k^{*} R}}\right|^{2}>\frac{v_{2} N_{0}}{a_{2} P_{B S}}\right)= \\
=\sum_{k=1}^{K}\left(\begin{array}{l}
K \\
k
\end{array}\right)(-1)^{k-1} \exp \left(-\frac{k v_{2} N_{0}}{a_{2} P_{B S} \lambda_{S R}}\right) .
\end{gathered}
$$

Next, (15) is used to calculate $\nabla_{2}$ as

$$
\begin{gathered}
\nabla_{2}=\operatorname{Pr}\left(\gamma_{D_{2}-x_{1}}>v_{2}, \gamma_{D_{2}-x_{2}}>v_{2}\right)= \\
=\operatorname{Pr}\left(\left|h_{R D_{2}}\right|^{2}>\frac{v_{2} N_{0}}{\left|h_{B R}\right|^{2} \xi\left(a_{1}-v_{2} a_{2}\right)},\right)= \\
\left.\left|h_{R D_{2}}\right|^{2}>\frac{v_{2} N_{0}}{a_{2} \xi\left|h_{B R}\right|^{2}}\right) \\
=\operatorname{Pr}\left(\left|h_{R D_{2}}\right|^{2}>\frac{v_{2} N_{0}}{\xi\left|h_{B R}\right|^{2}} \max \left(\frac{1}{\left(a_{1}-v_{2} a_{2}\right)}, \frac{1}{a_{2}}\right)\right) .
\end{gathered}
$$

To make our computation simpler, we denote $\theta=\max \left(\frac{1}{\left(a_{1}-v_{2} a_{2}\right)}, \frac{1}{a_{2}}\right), \nabla_{2}$ can be rewritten as

$$
\begin{gathered}
\nabla_{2}=\operatorname{Pr}\left(\left|h_{R D_{2}}\right|^{2}>\frac{\theta v_{2} N_{0}}{\xi\left|h_{B R}\right|^{2}}\right)= \\
=\int_{0}^{\infty}\left(1-F_{\left|h_{R D_{2}}\right|^{2}}\left(\frac{\theta v_{2} N_{0}}{\xi\left|h_{R D_{2}}\right|^{2}}\right)\right) f_{\left|h_{B R}\right|^{2}}(x) d x= \\
=\int_{0}^{\infty} \exp \left(-\frac{\theta v_{2} N_{0}}{\xi \lambda_{R D 2} x}\right) \frac{1}{\lambda_{B R}} \exp \left(-\frac{x}{\lambda_{B R}}\right) d x= \\
=\frac{1}{\lambda_{B R}} \int_{0}^{\infty} \exp \left(-\frac{\theta v_{2} N_{0}}{\xi \lambda_{R D 2} x}-\frac{x}{\lambda_{B R}}\right) d x= \\
=\sqrt{\frac{4 \theta v_{2} N_{0}}{\xi \lambda_{B R} \lambda_{R D 2}}} K_{1}\left(\sqrt{\frac{4 \theta v_{2} N_{0}}{\xi \lambda_{B R} \lambda_{R D 2}}}\right) .
\end{gathered}
$$

Replacing (B.1), (B.3) into (15), $\mathrm{OP}_{2}$ can be obtained.

This is end of the proof.

\section{APPENDIX C}

\section{Proof of the Proposition 3:}

Plugging (9) and (19) to corresponding result, $\psi_{1}$ can be written by

$$
\begin{gathered}
\psi_{1}=\operatorname{Pr}\left(\gamma_{S_{k^{*} R} R i p-x_{2}}>v_{2}\right)= \\
=\operatorname{Pr}\left(\mid h_{\left.S_{k^{*} R}\right|^{2}>} \frac{v_{2}\left(a_{1} P_{B S} \mid g_{\left.\left.S_{k^{*} R}\right|^{2}+N_{0}\right)}\right.}{a_{2} P_{B S}}\right)= \\
=\int_{0}^{\infty}\left(1-F_{\left|h_{S_{k^{*}} \mid}\right|^{2}}\left(\frac{v_{2}\left(a_{1} P_{B S} x+N_{0}\right)}{a_{2} P_{B S}}\right)\right) f_{\left|g_{S_{k^{*} *} \mid}\right|^{2}}(x) d x .
\end{gathered}
$$


Using (9) and (10), then further employs corresponding $\mathrm{CDF}$ and PDF, $\psi_{1}$ can be computed by

$$
\begin{gathered}
\psi_{1}=\sum_{k=1}^{K} \sum_{m=1}^{K}\left(\begin{array}{l}
K \\
k
\end{array}\right)\left(\begin{array}{c}
K \\
m
\end{array}\right)(-1)^{k+m-2} \frac{m}{\lambda_{\text {SRip }}} \times \\
\times \exp \left(-\frac{k v_{2} N_{0}}{a_{2} P_{B S} \lambda_{S R}}\right) \int_{0}^{\infty} \exp \left(-\left(\frac{k v_{2} a_{1}}{a_{2} \lambda_{S R}}+\frac{m}{\lambda_{\text {SRip }}}\right) x\right) d x= \\
=\sum_{k=1}^{K} \sum_{m=1}^{K}\left(\begin{array}{l}
K \\
k
\end{array}\right)\left(\begin{array}{l}
K \\
m
\end{array}\right)(-1)^{k+m-2} \frac{m a_{2} \lambda_{S R}}{k v_{2} a_{1} \lambda_{S R i p}+m a_{2} \lambda_{S R}} \times \\
\times \exp \left(-\frac{k v_{2} N_{0}}{a_{2} P_{B S} \lambda_{S R}}\right) .
\end{gathered}
$$

Based on (19), we compute $\psi_{2}$ as below

$$
\begin{gathered}
\psi_{2}=\operatorname{Pr}\left(\gamma_{D_{2} i p-x_{2}}>v_{2}\right)= \\
=\operatorname{Pr}\left(\left|h_{R D_{2}}\right|^{2}>\frac{v_{2}\left(a_{1} \xi\left|h_{B R}\right|^{2}\left|g_{R D_{2}}\right|^{2}+N_{0}\right)}{a_{2} \xi\left|h_{B R}\right|^{2}}\right)= \\
=\int_{0}^{\infty} \int_{0}^{\infty}\left(1-F_{\left|h_{R D_{2}}\right|^{2}}\left(\frac{v_{2}\left(a_{1} \xi x y+N_{0}\right)}{a_{2} \xi x}\right)\right) \times \\
\times f_{\left|h_{B R}\right|^{2}}(x) d x f_{\left|g_{R D_{2}}\right|^{2}}(y) d y .
\end{gathered}
$$

In next step, using results of $\mathrm{CDF}$ and $\mathrm{PDF}, \psi_{2}$ is formulated as

$$
\begin{gathered}
\psi_{2}=\frac{1}{\lambda_{B R}} \frac{1}{\lambda_{R D 2 i p}} \int_{0}^{\infty} \exp \left(-\left(\frac{v_{2} a_{1} \xi}{a_{2} \xi \lambda_{R D 2}}+\frac{1}{\lambda_{R D 2 i p}}\right) y\right) d y \times \\
\times \int_{0}^{\infty} \exp \left(-\frac{v_{2} N_{0}}{a_{2} \xi \lambda_{R D 2} x}-\frac{x}{\lambda_{B R}}\right) d x= \\
=\frac{a_{2} \xi \lambda_{R D 2}}{v_{2} a_{1} \xi \lambda_{R D 2 i p}+a_{2} \xi \lambda_{R D 2}} \times \\
\times \sqrt{\frac{4 v_{2} N_{0}}{a_{2} \xi \lambda_{R D 2} \lambda_{B R}}} K_{1}\left(\sqrt{\frac{4 v_{2} N_{0}}{a_{2} \xi \lambda_{R D 2} \lambda_{B R}}}\right)
\end{gathered}
$$

Plugging (C.2), (C.4) into (19), $O P_{2 i p}$ can be achieved.

This completes the proof.

\section{CONFLICTS OF INTEREST}

The authors declare that they have no conflicts of interest.

\section{REFERENCES}

[1] L. Chettri and R. Bera, "A comprehensive survey on Internet of Things (IoT) toward 5G wireless systems", IEEE Internet of Things Journal, vol. 7, no. 1, pp. 16-32, Jan. 2020. DOI: 10.1109/JIOT.2019.2948888.

[2] T. Lv, Y. Ma, J. Zeng, and P. T. Mathiopoulos, "Millimeter-wave NOMA transmission in cellular M2M communications for Internet of Things", IEEE Internet of Things Journal, vol. 5, no. 3, pp. 19892000, Jun. 2018. DOI: 10.1109/JIOT.2018.2819645.

[3] L. P. Qian, B. Shi, Y. Wu, B. Sun, and D. H. K. Tsang, "NOMA- enabled mobile edge computing for Internet of Things via joint communication and computation resource allocations", IEEE Internet of Things Journal, vol. 7, no. 1, pp. 718-733, Jan. 2020. DOI: 10.1109/JIOT.2019.2952647.

[4] S. Han et al., "Energy efficient secure computation offloading in NOMA-based mMTC networks for IoT", IEEE Internet of Things Journal, vol. 6, no. 3, pp. 5674-5690, Jun. 2019. DOI: 10.1109/JIOT.2019.2904741.

[5] V. Markevicius, D. Navikas, D. Andriukaitis, M. Cepenas, A Valinevicius, M. Zilys, R. Malekian, A. Janeliauskas, W. Walendziuk, A. Idzkowski, "Two thermocouples low power wireless sensors network", AEU - International Journal of Electronics and Communications, vol. 84, pp. 242-250, 2018. DOI: 10.1016/j.aeue.2017.11.032.

[6] A. Rauniyar, D. H. Hagos, and M. Shrestha, "A crowd-based intelligence approach for measurable security, privacy, and dependability in internet of automated vehicles with vehicular fog", Mobile Inf. Syst., vol. 2018, article ID 7905960, Jan. 2018. DOI: $10.1155 / 2018 / 7905960$

[7] M. Wollschlaeger, T. Sauter, and J. Jasperneite, "The future of industrial communication: Automation networks in the era of the Internet of Things and industry 4.0", IEEE Ind. Electron. Mag., vol. 11, no. 1, pp. 17-27, Mar. 2017. DOI: 10.1109/MIE.2017.2649104.

[8] Q. Qi and X. Chen, "Wireless powered massive access for cellular Internet of Things with imperfect SIC and nonlinear EH", IEEE Internet of Things Journal, vol. 6, no. 2, pp. 3110-3120, Apr. 2019. DOI: 10.1109/JIOT.2018.2878860

[9] X. Lu, P. Wang, D. Niyato, D. I. Kim, and Z. Han, "Wireless networks with RF energy harvesting: A contemporary survey", IEEE Commun. Surveys Tuts., vol. 17, no. 2, pp. 757-789, secondquarter 2015. DOI: $10.1109 /$ COMST.2014.2368999.

[10] D.-T. Do, "Energy-aware two-way relaying networks under imperfect hardware: Optimal throughput design and analysis", Telecommunication Systems (Springer), vol. 62, no. 2, pp. 449-459, 2015. DOI: $10.1007 / \mathrm{s} 11235-015-0085-7$.

[11] M. Wakaiki, K. Suto, K. Koiwa, K. Liu, and T. Zanma, "A controltheoretic approach for cell zooming of energy harvesting small cell networks", IEEE Trans. on Green Communications and Networking, vol. 3, no. 2, pp. 329-342, Jun. 2019. DOI: 10.1109/TGCN.2018.2889897.

[12] H. Wang, J. Wang, G. Ding, L. Wang, T. A. Tsiftsis, and P. K. Sharma, "Resource allocation for energy harvesting-powered D2D communication underlaying UAV-assisted networks", IEEE Trans. on Green Communications and Networking, vol. 2, no. 1, pp. 14-24, Mar. 2018. DOI: 10.1109/TGCN.2017.2767203.

[13] M. Pratibha, K. H. Li, and K. C. Teh, "Channel selection in multichannel cognitive radio systems employing RF energy harvesting", IEEE Trans. Veh. Technol., vol. 65, no. 1, pp. 457-462, Jan. 2016. DOI: 10.1109/TVT.2015.2392770.

[14] J. Ren, J. Hu, D. Zhang, H. Guo, Y. Zhang, and X. Shen, "RF energy harvesting and transfer in cognitive radio sensor networks: Opportunities and challenges", IEEE Commun. Mag., vol. 56, no. 1, pp. 104-110, Jan. 2018. DOI: 10.1109/MCOM.2018.1700519.

[15] L. Dai, B. Wang, Z. Ding, Z. Wang, S. Chen, and L. Hanzo, "A survey of non-orthogonal multiple access for 5G", IEEE Communications Surveys \& Tutorials, vol. 20, no. 3, pp. 2294-2323, thirdquarter 2018. DOI: 10.1109/COMST.2018.2835558.

[16] T.-L. Nguyen and Dinh-Thuan Do, "Power allocation schemes for wireless powered NOMA systems with imperfect CSI: An application in multiple antenna-based relay," International Journal of Communication Systems, vol. 31, no. 15, e3789, 2018. DOI: 10.1002/dac.3789..

[17] D.-T. Do and A.-T. Le, "NOMA based cognitive relaying: Transceiver hardware impairments, relay selection policies and outage performance comparison", Computer Communications, vol. 146, pp. 144-154, 2019. DOI: 10.1016/j.comcom.2019.07.023.

[18] Dinh-Thuan Do, M.-S. V. Nguyen, T. N. Nguyen, X. Li and K. Choi, "Enabling Multiple Power Beacons for Uplink of NOMA-Enabled Mobile Edge Computing in Wirelessly Powered IoT," IEEE Access, vol. $\quad 8, \quad$ pp. $148892-148905, \quad 2020 . \quad$ DOI: 10.1109/ACCESS.2020.3015741.

[19] I. S. Gradshteyn and I. M. Ryzhik, Table of Integrals, Series and Products, 6th ed. New York: Academic Press, 2000. 